\title{
Boundary vibration control of a floating wind turbine system with mooring lines ${ }^{\text {皿 }}$
}

\author{
Wei He ${ }^{\mathrm{a}, \mathrm{b}, *}$, Weijie Xiang a,b ${ }^{\mathrm{a}}$, Xiuyu He $\mathrm{He}^{\mathrm{a}, \mathrm{b}, \mathrm{c}}$, Guang $\mathrm{Li}^{\mathrm{d}}$ \\ ${ }^{a}$ Institute of Artificial Intelligence, University of Science and Technology Beijing, Beijing 100083, China \\ ${ }^{\mathrm{b}}$ School of Automation and Electrical Engineering, University of Science and Technology Beijing, Beijing 100083, China \\ ' Shunde Graduate School, University of Science and Technology Beijing, Foshan, 528399, China \\ d School of Engineering and Materials Science, Queen Mary University of London, Mile End Road, London E1 4Ns, UK
}

\section{A R T I C L E I N F O}

\section{Keywords:}

Floating wind turbine

Mooring lines

Distributed parameter system (DPS)

Vibration control

\begin{abstract}
A B S T R A C T
In this paper, we investigate dynamic modeling, active boundary control design, and stability analysis for a coupled floating wind turbine (FWT) system, which is connected with two flexible mooring lines. It is a coupled beam-strings structure, and we design two boundary controllers to restrain the vibrations of this flexible system caused by external disturbances based on the coupled partial differential equations and ordinary differential equations (PDEs-ODEs) model. Meanwhile, significant performance of designed boundary controllers and system's stability are theoretically analyzed, and a set of simulation results are provided to show efficacy of the proposed approach.
\end{abstract}

\section{Introduction}

The offshore area has abundant wind energy resource, especially in deep water area $(>100 \mathrm{~m})$, and floating platforms are widely used in energy exploitation in offshore engineering. In offshore area, floating wind turbine (FWT) system, Fig. 1, is a large wind energy conversion equipment including wind generator tower, floating platform, and mooring lines. It is suitable for a harsh deep-sea environment where the bottom-mounted tower is infeasible (Lamas \& Fernandez, 2011). However, the complex marine environment has a great impact on FWT system, and turbine tower and mooring lines will generate wave-induced oscillation, which may cause irreversible damage and premature fatigue. Thus, for the conversion efficiency and safety concern, structural vibrations of FWT system need to be studied and addressed.

In recent years, many works focus on vibration control design and dynamic analysis for the FWT systems. In previous works, many researchers use passive control methods to address the vibration reduction of the system, In general, passive control is a control without external energy, and the control force is generated passively by vibration deformation of the control device along the structure or the movement of the device itself. Common passive control includes tuned mass damper (De Domenico \& Ricciardi, 2018; Stewart \& Lackner,
2013; Tong, Zhao, \& Zhao, 2017), tuned liquid damper (Fu, Jiang, \& Wu, 2019; Zhang, Basu, \& Nielsen, 2019), friction damper, etc. In Si, Karimi, and Gao (2013), the OC3-Hywind FWT system is discussed with a passive structural controller. Another vibration is active control which is to use external energy to exert the control force and the control device can use external energy to tune its own parameters for adjusting control force. Therefore, active control may have a better performance than passive control. In Bakka, Karimi, and Christiansen (2014), the authors analyze dynamic behavior of an offshore wind turbine system by considering linear varying parameter and constrained information, and establish a dynamic model. In Bakka and Karimi (2013), an outputfeedback control method for an offshore FWT system with constraint is proposed to reduce wave-oscillations. In Bakka and Karimi (2012), many kinds of linear models are introduced to represent a wind turbine system. In addition, a robust output feedback controller integration using $H_{\infty}$ control with pole setting constraints is investigated for a wind turbine system. In Li and Gao (2016), the authors propose a novel $H_{\infty}$ control method based on a linear model and apply it to an FWT system. These works make great achievements in the modeling and control of the FWT system. However, they analyze dynamics of the FWT and address the control problem based on the linear or finitedimensional model because of regarding the wind turbine tower as a

\footnotetext{
This work was supported in part by the National Key Research and Development Program of China under Grant 2019YFB1703600, the National Natural Science Foundation of China under Grant 61873298, the Newton Advanced Fellowship from The Royal Society, UK, under Grant NA160436, the China Postdoctoral Science Foundation under Grant 2019TQ0029. This work was also supported by Beijing Top Discipline for Artificial Intelligent Science and Engineering, University of Science and Technology Beijing.

* Corresponding author.

E-mail addresses: weihe@ieee.org (W. He), xiangweijie666@gmail.com (W. Xiang), xiuyuhe@ieee.org (X. He), g.li@qmul.ac.uk (G. Li).
} 

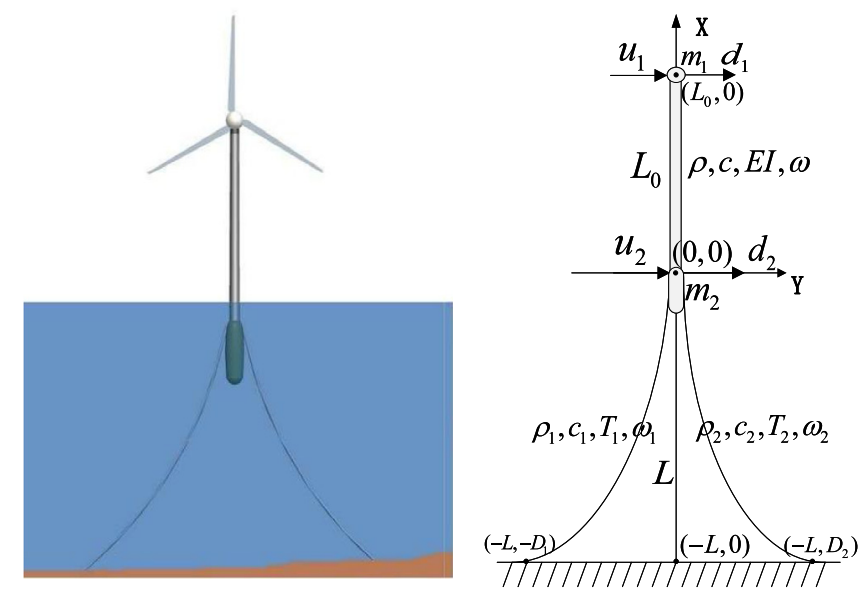

Fig. 1. The floating wind turbine system.

rigid body system. From the structure of FWT, it is a coupled beamstrings system which is characterized with infinite modes (Guo \& Jin, 2015; Ren, Wang, \& Krstic, 2013; Wu \& Wang, 2014; Wu, Wang, \& Li, 2012), namely, using PDEs to describe the system's dynamics. In this work, we consider the FWT system as a flexible coupled structure and address the vibration suppression problem of the turbine tower and mooring lines.

For vibration reduction of flexible structures, many vibration control methods are developed (Bhikkaji, Moheimani, \& Petersen, 2012; Chen \& Chang, 2013; Do, 2017; He, He, Shi \& Sun, 2017; He, He \& Sun, 2017; Jin \& Guo, 2015; Liu, He, Zhao, Ahn, \& Li, 2020; Pota \& Alberts, 1995), e.g., adaptive control method (Chao, Chenguang, \& Zhaopeng, 2020; Chen, Shao, \& Jiang, 2017; Dai, He, Wang, \& Yuan, 2018; He, He, Liu, Wang, Li, \& Wang, 2018; Li, Chen, Fu \& Sun, 2016; Li et al., 2017; Li, Wang, Du \& Boulkroune, 2016; Ren, Chen, \& Liu, 2020; Xie, Sun, Wen, Hei, \& Qian, 2019), optimal control method (Sun \& Xia, 2009; Wu, Sun, \& Chen, 2018) and sliding mode control method (Wang, Liu, Ren, \& Chen, 2015), force control method (Endo, Sasaki, \& Matsuno, 2017; Endo, Sasaki, Matsuno, \& Jia, 2016), etc., are also investigated. Compared with rigid structure (Li, Chen, Wang, Zhang, \& Wang, 2019; Ming, Yanlu, Huifang, \& Junzhi, 2020), flexible structures has the advantages of light weight and strong flexibility. In Moheimani, Pota, and Petersen (1999), a novel spatial balanced model is established for a flexible pinned-pinned beam, and vibration control is proposed based on the system model. In Bhikkaji et al. (2012), the vibration control problem of a flexible beam with collocated sensor/actuator pairs is investigated. Notice that some flexible systems mentioned above are discretized as a truncated model, namely considering finite modes of the system. Furthermore, the control design for flexible systems is conducted based on the discretized model. However, these unmodeled modes, including high-frequency modes, may have a great impact on the system performance, and it is valuable to consider the influences of these modes.

Boundary control is a control method which considers the dynamics of entire modes and has been applied to various infinite-dimensional systems widely, e.g., heat equations (Huang, Xu, Li, Xu, \& Yu, 2013; Wang, Ren, \& Krstic, 2012), moving string system (He, Ge, \& Huang, 2015; Nguyen \& Hong, 2010, 2012), etc. For the wind turbine tower system, there exist some literature to address the vibration reduction problem of the tower based on boundary control strategy. A nonlinear vibration isolator is proposed to reduce the structural impact of turbine under seismal loading and wind in Van der Woude (2011), which is a novelty work used in the wind turbine. In He and Ge (2015), a vibration controller is investigated for a nonuniform wind turbine tower with random wind loads. However, these works consider the dynamics of a single flexible structure and focus on the vibration suppression of wind turbine tower. In this work, we consider the vibration control of FWT system, which is a coupled beam-strings system. The main challenges are to consider the strong coupling between turbine tower and mooring lines, and to develop boundary controllers to reduce vibrations of FWT system. For mooring systems, motions of the mooring lines are usually regarded as an external force acted to floating platforms in the previous works. In Chen, Ge, How, and Choo (2013), Tee and Ge (2006), the authors study dynamics of mooring lines by ignoring the connection of the mooring lines and the ship. Nevertheless, the coupling relationship has a great influence on the performance of mooring system. Notice that we will consider the strong coupling between mooring lines, floating platform, and flexible turbine tower, and establish a nonlinear dynamic model. Furthermore, we will propose two boundary controllers simultaneously to reduce the vibration of flexible turbine tower and mooring lines and to compensate for the performance degradation.

In brief, main works of this paper are summarized as

(i) A coupled beam-strings model is used to illustrate the dynamical behavior of FWT system, and the coupled relationship among turbine tower, floating platform, and mooring lines is considered in dynamic analysis. A coupled hybrid PDE-ODEs model is established to describe FWT system by applying Hamilton's principle.

(ii) Two boundary controllers are designed to ensure the stability of the system simultaneously based on nonlinear coupled model under unknown environment loads. Theoretical analysis and simulation results are presented to demonstrate the effectiveness of proposed controllers.

The rest of this paper are summarized as follows. In Section 2, we establish the system model described by PDEs using Hamilton's principle, to derive the governing equations (described by PDEs) and boundary conditions (described by ODEs) of FWT system. In Section 3, the control objectives and uniform boundedness of the closed-loop system are derived by Lyapunov's direct method. In Section 4, simulation results demonstrate the efficacy of the proposed approach. In Section 5, we give conclusions of this paper and future works.

\section{System modeling and preliminaries}

As shown in Fig. 1, FWT system is described by a coupled beamstrings structure, where, the turbine tower is regarded as a flexible beam with a lumped-mass-like payload, and mooring lines are regarded as two coupled strings (Zhang, Yang, Nie, \& He, 2015). The control force $u_{1}(t)$ is mounted on the top of tower and the control force $u_{2}(t)$ is mounted on the floating platform. $L_{0}$ and $L$ are the tower's and mooring lines' length, $m_{1}$ and $m_{2}$ are the mass of top of tower and floating platform, respectively. The mass per unit length of the tower is $\rho$. $D_{1}$ and $D_{2}$ are original locations of mooring lines. $\rho_{1}$ and $\rho_{2}$ are the mass per unit length of mooring lines, respectively. $c, c_{1}$ and $c_{2}$ are the damping coefficients of flexible tower and mooring lines. $w_{1}$ represents the displacement of left line, and $w_{2}$ represents the displacement of right line. $w$ represents the displacement of wind turbine tower. Moreover, EI represents the bending stiffness of beamlike tower, and tensions of two mooring lines are expressed as $T_{1}$ and $T_{2}$, respectively. $X-Y$ coordinate system is a plane coordinate system, where, $X$ is used to determine the position and $Y$ is the displacement of mooring lines and flexible wind tower. Meanwhile, floating platform is fixed on the sea level by mooring lines, its position will not follow the FWT system.

Remark 1. For conciseness, the notations $w^{\prime \prime}=\partial^{2} w / \partial x^{2}, \ddot{w}=$ $\partial^{2} w / \partial t^{2}, w^{\prime}=\partial w / \partial x, \dot{w}=\partial w / \partial t, w=w(x, t), w_{L_{0}}=w\left(L_{0}, t\right)$ and $w_{0}=w(0, t)$ are used. 


\subsection{Dynamical model of floating wind turbine vibrations}

The FWT system's kinetic energy $E_{k}(t)$ can be obtained as

$$
\begin{aligned}
E_{k}(t)= & \frac{\rho_{1}}{2} \int_{-L}^{0}\left(\frac{\partial\left[w_{1}+D_{1}\right]}{\partial t}\right)^{2} d x+\frac{\rho_{2}}{2} \int_{-L}^{0}\left(\frac{\partial\left[w_{2}-D_{2}\right]}{\partial t}\right)^{2} d x \\
& +\frac{\rho}{2} \int_{0}^{L_{0}}\left(\frac{\partial w}{\partial t}\right)^{2} d x+\frac{m_{1}}{2}\left(\frac{\partial w\left(L_{0}, t\right)}{\partial t}\right)^{2}+\frac{m_{2}}{2}\left(\frac{\partial w(0, t)}{\partial t}\right)^{2},
\end{aligned}
$$

and the potential energy $E_{p}(t)$ can be written as

$$
\begin{aligned}
E_{p}(t)= & \frac{E I}{2} \int_{0}^{L_{0}}\left(\frac{\partial^{2} w}{\partial x^{2}}\right)^{2} d x+\frac{T_{1}}{2} \int_{-L}^{0}\left(\frac{\partial\left[w_{1}+D_{1}\right]}{\partial x}\right)^{2} d x \\
& +\frac{T_{2}}{2} \int_{-L}^{0}\left(\frac{\partial\left[w_{2}-D_{2}\right]}{\partial x}\right)^{2} d x
\end{aligned}
$$

where, $t$ and $x$ denote the time variable and the position variable, respectively.

Remark 2. From Fig. 1, because the strong coupling of the two flexible structures, we have $w=w_{1}=w_{2}$ at $x=0$ and $\partial w / \partial t=\partial w_{1} / \partial t=\partial w_{1} / \partial t$ at $x=0$.

The virtual work done by the model is

$$
\begin{aligned}
\delta W_{c}(t)= & -c \int_{0}^{L} \frac{\partial w}{\partial t} \delta w d x-c_{1} \int_{0}^{L} \frac{\partial\left[w_{1}+D_{1}\right]}{\partial t} \delta w_{1} d x \\
& -c_{2} \int_{0}^{L} \frac{\partial\left[w_{2}-D_{2}\right]}{\partial t} \delta w_{2} d x .
\end{aligned}
$$

where the FWT system damping coefficients are represented as $c, c_{1}$ and $c_{2}$.

The virtual work done by external disturbances $d_{1}(t)$ and $d_{2}(t)$ is obtained by

$\delta W_{d}(t)=d_{1}(t) \delta w\left(L_{0}, t\right)+d_{2}(t) \delta w(0, t)$.

Remark 3. The lumped mass at the top boundary of the tower suffers from the boundary disturbance $d_{1}(t) . d_{1}(t)$ is the synthesis of all disturbances to the components at the tip boundary of tower. $d_{2}(t)$ is the wave disturbance mainly about waves with different height, speed etc., for example, the wave with significant wave height and the theoretical wave height (Ainsworth \& Juneau, 2006; Holthuijsen, 2007) that can result in large excitation forces. All these excitation forces acting on the FWT system as external disturbances can cause vibrations of tower.

The virtual work $W_{u}(t)$ done by the controllers $u_{1}(t)$ and $u_{2}(t)$ is denoted as

$\delta W_{u}(t)=u_{1}(t) \delta w\left(L_{0}, t\right)+u_{2}(t) \delta w(0, t)$.

Then, the entire virtual work $W(t)$ done on the system can be represented as

$\delta W(t)=\delta W_{c}(t)+\delta W_{d}(t)+\delta W_{u}(t)$.

According to the Hamilton's principle (Goldstein, 1951), governing equations of the FWT system including the turbine tower and mooring lines are obtained as

$$
\begin{aligned}
& \rho \ddot{w}+E I w^{\prime \prime \prime \prime}+c \dot{w}=0, \quad(x, t) \in\left(0, L_{0}\right) \times[0, \infty) . \\
& \rho_{i} \ddot{w}_{i}-T_{i} w_{i}^{\prime \prime}+c_{i} \dot{w}_{i}=0, i=(1,2) \text { and }(x, t) \in(-L, 0) \times[0, \infty) .
\end{aligned}
$$

with boundary conditions

$$
\begin{aligned}
w_{0}^{\prime \prime} & =w_{L_{0}}^{\prime \prime}=0, \\
m_{1} \ddot{w}_{L_{0}}-E I w_{L_{0}}^{\prime \prime \prime} & =u_{1}(t)+d_{1}(t) \\
w_{1}(-L, t) & =-D_{1}, \\
w_{2}(-L, t) & =D_{2},
\end{aligned}
$$

$m_{2} \ddot{w}_{0}+E I w_{0}^{\prime \prime \prime}+T_{1} w_{1}^{\prime}(0, t)+T_{2} w_{2}^{\prime}(0, t)=u_{2}(t)+d_{2}(t)$.

$\forall t \in[0, \infty)$.

Remark 4. The governing equations (7) and (8) describe the dynamics of flexible structures including two mooring lines and a flexible tower. Meanwhile, the boundary conditions (9), (11), and (12) describe the dynamics of the boundary position of flexible structures and they satisfy the Newton's second law From the form of these equations, $\ddot{w}_{0}$ and $\ddot{w}_{L_{0}}$ are the acceleration of floating platform and top of tower. A part of boundary conditions is nonconservative force which includes that $u_{2}(t)$ and $u_{1}(t)$ are boundary control forces acting on platform and top of tower, and $d_{2}(t)$ and $d_{1}(t)$ are external boundary disturbances. $E I w_{L_{0}}^{\prime \prime \prime}$, $E I w_{0}^{\prime \prime \prime}$ and $T_{1} w_{1}^{\prime}(0, t), T_{2} w_{2}^{\prime}(0, t)$ are strain forces of two boundary of flexible tower and tensions of two mooring lines. From Eq. (12), it is easy known that the motion of platform is related to the dynamics of mooring lines and flexible tower, and the coupling relation has an effect on the dynamic modeling of FWT system.

Remark 5. For the FWT system, the distributed disturbances have less influence on the dynamics of the flexible system comparing with the boundary disturbances. In this paper, we ignore the effect of disturbances for its less influence and simplifying the theoretical analysis.

\subsection{Preliminaries}

For stability analysis, we present lemmas, remark, and assumption for the subsequent development as follows.

Lemma 1 (He, Zhang, \& Ge, 2014). For any continuously differentiable $\phi$ on $\left[L_{1}, L_{2}\right]$, we have

$\left[\phi-\phi\left(L_{1}, t\right)\right]^{2} \leq\left(L_{2}-L_{1}\right) \int_{L_{1}}^{L_{2}}\left[\phi^{\prime}\right]^{2} d x$.

Lemma 2 (He et al., 2014). For any continuously differentiable $\phi$ on $\left[L_{1}, L_{2}\right]$, we have

$[\phi]^{2} \leq 2\left(L_{2}-L_{1}\right) \int_{L_{1}}^{L_{2}}\left[\phi^{\prime}\right]^{2} d x+2\left[\phi\left(L_{1}, t\right)\right]^{2}$.

Lemma 3 (Poincaré Inequality Smyshlyaev and Krstic (2010)). For any $\phi$, continuously differentiable on $\left[L_{1}, L_{2}\right]$, we have

$\int_{L_{1}}^{L_{2}}[\phi]^{2} d x \leq 2\left(L_{2}-L_{1}\right) \phi^{2}\left(L_{2}, t\right)+4\left(L_{2}-L_{1}\right)^{2} \int_{L_{1}}^{L_{2}}\left[\phi^{\prime}\right]^{2} d x$,

$\int_{L_{1}}^{L_{2}}[\phi]^{2} d x \leq 2\left(L_{2}-L_{1}\right) \phi^{2}\left(L_{1}, t\right)+4\left(L_{2}-L_{1}\right)^{2} \int_{L_{1}}^{L_{2}}\left[\phi^{\prime}\right]^{2} d x$.

Remark 6. From Poincaré inequality (16), we further have

$[\phi]^{2} \leq 4\left(L_{2}-L_{1}\right)^{2} \phi^{\prime 2}\left(L_{1}\right)+8\left(L_{2}-L_{1}\right)^{3} \int_{L_{1}}^{L_{2}}\left[\phi^{\prime \prime}\right]^{2} d x+2\left[\phi\left(L_{1}\right)\right]^{2}$.

Assumption 1. According to the unknown external boundary disturbances $d_{1}(t)$ and $d_{2}(t)$, we assume that the two positive values $\bar{d}_{1} \in \mathbb{R}^{+}$ and $\bar{d}_{2} \in \mathbb{R}^{+}$exist, to make $\left|d_{1}(t)\right| \leq \bar{d}_{1}$ and $\left|d_{2}(t)\right| \leq \bar{d}_{2}$ hold. This assumption is appropriate because the disturbances have finite energy.

\section{Controller design}

In this part, we design controllers $u_{1}(t)$ and $u_{2}(t)$ for vibrations suppression of the FWT system under external disturbances.

Theorem 1. Based on the dynamic model (7) - (8) and (9)-(13), the boundary controllers are proposed as follow

$u_{1}(t)=-k_{1}\left[\dot{w}_{L_{0}}+\frac{\alpha}{\beta} w_{L_{0}}\right]-\operatorname{sgn}\left[\dot{w}_{L_{0}}+\frac{\alpha}{\beta} w_{L_{0}}\right] \bar{d}_{1}-\frac{\alpha}{\beta} m_{1} \dot{w}_{L_{0}}$, 
$u_{2}(t)=-k_{2}\left[\dot{w}_{0}+\frac{\alpha}{\beta} w_{0}\right]-k_{p} w_{0}-\operatorname{sgn}\left[\dot{w}_{0}+\frac{\alpha}{\beta} w_{0}\right] \bar{d}_{2}-\frac{\alpha}{\beta} m_{2} \dot{w}_{0}$,

where $k_{1}, k_{2}$ and $k_{p}$ are three positive control gains, $\alpha$ and $\beta$ are the positive constants, and $\operatorname{sgn}(\cdot)$ represents the signum function. The closed-loop system is uniformly bounded as the initial conditions are bounded.

Proof. Consider the Lyapunov candidate function as

$\Theta(t)=\Theta_{1}(t)+\Theta_{2}(t)+\Theta_{3}(t)+\Delta(t)$,

where $\Theta_{1}(t), \Theta_{2}(t), \Theta_{3}(t)$ and $\Delta(t)$ are defined as

$$
\begin{aligned}
\Theta_{1}(t)= & \frac{\beta}{2} \int_{0}^{L_{0}} \rho[\dot{w}]^{2} d x+\frac{\beta}{2} E I \int_{0}^{L_{0}}\left[w^{\prime \prime}\right]^{2} d x \\
& +\frac{\beta}{2} \int_{-L}^{0}\left(\rho_{1}\left[\dot{w}_{1}\right]^{2}+\rho_{2}\left[\dot{w}_{2}\right]^{2}\right) d x \\
& +\frac{\beta}{2} \int_{-L}^{0}\left(T_{1}\left[w_{1}^{\prime}\right]^{2}+T_{2}\left[w_{2}^{\prime}\right]^{2}\right) d x \\
\Theta_{2}(t)= & \frac{\beta}{2} m_{1}\left[\dot{w}_{L_{0}}+\frac{\alpha}{\beta} w_{L_{0}}\right]^{2}+\frac{\beta}{2} m_{2}\left[\dot{w}_{0}+\frac{\alpha}{\beta} w_{0}\right]^{2}+\frac{\beta}{2} k_{p}\left[w_{0}\right]^{2}, \\
\Theta_{3}(t)= & \frac{\alpha}{2} \int_{0}^{L_{0}} c[w]^{2} d x+\frac{\alpha}{2} \int_{-L}^{0} c_{1}\left[w_{1}\right]^{2} d x+\frac{\alpha}{2} \int_{-L}^{0} c_{2}\left[w_{2}\right]^{2} d x, \\
\Delta(t)= & \alpha \int_{0}^{L_{0}} \rho \dot{w} w d x+\alpha \int_{-L}^{0}\left[\rho_{1} \dot{w}_{1} w_{1}+\rho_{2} \dot{w}_{2} w_{2}\right] d x \\
& -\gamma \int_{-L}^{0} x\left[\rho_{1} \dot{w_{1}} w_{1}^{\prime}+\rho_{2} \dot{w}_{2} w_{2}^{\prime}\right] d x
\end{aligned}
$$

where $\gamma$ is a positive weighting constant.

Consider a positive function $\kappa(t)$ as

$$
\begin{aligned}
\kappa(t)= & \int_{0}^{L_{0}}\left\{[\dot{w}]^{2}+[w]^{2}+\left[w^{\prime \prime}\right]^{2}\right\} d x+\int_{-L}^{0}\left\{\left[\dot{w}_{1}\right]^{2}+\left[w_{1}\right]^{2}+\left[w_{1}^{\prime}\right]^{2}\right. \\
& \left.+\left[\dot{w}_{2}\right]^{2}+\left[w_{2}\right]^{2}+\left[w_{2}^{\prime}\right]^{2}\right\} d x .
\end{aligned}
$$

From the expression of $\Theta_{1}(t)$ and $\Theta_{3}(t)$, we have

$\mu_{1} \kappa(t) \leq \Theta_{1}(t)+\Theta_{3}(t) \leq \mu_{2} \kappa(t)$,

where

$\mu_{1}=\min \left(\frac{\beta \rho}{2}, \frac{\beta E I}{2}, \frac{\alpha c}{2}, \frac{\beta \rho_{1}}{2}, \frac{\beta T_{1}}{2}, \frac{\alpha c_{1}}{2}, \frac{\beta \rho_{2}}{2}, \frac{\beta T_{2}}{2}, \frac{\alpha c_{2}}{2}\right)$,

$\mu_{2}=\max \left(\frac{\beta \rho}{2}, \frac{\beta E I}{2}, \frac{\alpha c}{2}, \frac{\beta \rho_{1}}{2}, \frac{\beta T_{1}}{2}, \frac{\alpha c_{1}}{2}, \frac{\beta \rho_{2}}{2}, \frac{\beta T_{2}}{2}, \frac{\alpha c_{2}}{2}\right)$.

From the expression of $\Delta(t)$, we know that $\Delta(t)$ is bounded as $|\Delta(t)| \leq$ $\mu_{3} \kappa(t)$, where $\mu_{3}=\max \left(\alpha \rho, \alpha \rho_{1}+\gamma L \rho_{1}, \alpha \rho_{2}+\gamma L \rho_{2}\right)$.

Considering $\alpha, \beta$ and $\gamma$ satisfying $\mu_{1}-\mu_{3}>0$, we have

$0<\mu_{4}\left[\Theta_{1}(t)+\Theta_{3}(t)\right] \leq \Theta_{1}(t)+\Theta_{3}(t)+\Delta(t) \leq \mu_{5}\left[\Theta_{1}(t)+\Theta_{3}(t)\right]$,

where $\mu_{4}=\frac{\mu_{1}-\mu_{3}}{\mu_{2}}$, and $\mu_{5}=\frac{\mu_{2}+\mu_{3}}{\mu_{1}}$. Then the Lyapunov candidate function is bounded as

$\lambda_{1}\left[\Theta_{1}(t)+\Theta_{2}(t)+\Theta_{3}(t)\right] \leq \Theta(t) \leq \lambda_{2}\left[\Theta_{1}(t)+\Theta_{2}(t)+\Theta_{3}(t)\right]$,

where $\lambda_{1}=\min \left(\mu_{4}, 1\right), \lambda_{2}=\max \left(\mu_{5}, 1\right)$.

Lemma 4. With above assumptions and defines, the time derivative of Lyapunov candidate function (21) is bounded with

$\dot{\Theta}(t) \leq-\lambda \Theta(t)+\varepsilon$,

where $\lambda$ and $\varepsilon$ are two positive constants.

Proof. Differentiation $\Theta(t)$ leads to

$\dot{\Theta}(t)=\dot{\Theta}_{1}(t)+\dot{\Theta}_{2}(t)+\dot{\Theta}_{3}(t)+\dot{\Delta}(t)$,

From Remark 2 and boundary condition (10), using Lamma 3, and substituting the proposed control (19) and (20), and let $\gamma L-\alpha \geq 0$, we further have

$\dot{\Theta}(t) \leq-\beta k_{1}\left[\dot{w}_{L_{0}}+\frac{\alpha}{\beta} w_{L_{0}}\right]^{2}-\beta k_{2}\left[\dot{w}_{0}+\frac{\alpha}{\beta} w_{0}\right]^{2}$

$$
\begin{aligned}
& -\left(\alpha k_{p}-\sigma_{1}-\sigma_{2}-\sigma_{3}\right)\left[w_{0}\right]^{2} \\
& -(\beta c-\alpha \rho) \int_{0}^{L_{0}}[\dot{w}]^{2} d x-\left(\alpha E I-8 \sigma_{1} L_{0}^{3}\right) \int_{0}^{L_{0}}\left[w^{\prime \prime}\right]^{2} d x \\
& -\left(\beta c_{1}-\alpha \rho_{1}-\frac{\gamma c_{1} L}{2}-\frac{\gamma}{2} \rho_{1}\right) \int_{-L}^{0}\left[\dot{w}_{1}\right]^{2} d x \\
& -\left(\beta c_{2}-\alpha \rho_{2}-\frac{\gamma c_{2} L}{2}-\frac{\gamma}{2} \rho_{2}\right) \int_{-L}^{0}\left[\dot{w}_{2}\right]^{2} d x \\
& -\left(\alpha T_{1}-\frac{\gamma}{2} T_{1}-\frac{\gamma c_{1} L}{2}-2 \sigma_{2} L\right) \int_{-L}^{0}\left[w_{1}^{\prime}\right]^{2} d x \\
& -\left(\alpha T_{2}-\frac{\gamma}{2} T_{2}-\frac{\gamma c_{2} L}{2}-2 \sigma_{3} L\right) \int_{-L}^{0}\left[w_{2}^{\prime}\right]^{2} d x-\frac{\sigma_{1}}{2 L_{0}} \int_{0}^{L_{0}} w^{2} d x \\
& -\frac{\sigma_{2}}{2 L} \int_{-L}^{0} w_{1}^{2} d x-\frac{\sigma_{3}}{2 L} \int_{-L}^{0} w_{2}^{2} d x+\frac{\alpha T_{1}}{2} D_{1}^{2}+\frac{\alpha T_{2}}{2} D_{2}^{2} .
\end{aligned}
$$

where $\sigma_{1}, \sigma_{2}$ and $\sigma_{3}$ are positive constants. Then, we have

$\dot{\Theta}(t) \leq-\lambda_{3}\left[\Theta_{1}(t)+\Theta_{2}(t)+\Theta_{3}(t)\right]+\varepsilon$,

where $\varepsilon=\frac{\alpha T_{1}}{2} D_{1}^{2}+\frac{\alpha T_{2}}{2} D_{2}^{2}$, and

$$
\begin{aligned}
\epsilon_{1}= & \beta c-\alpha \rho>0, \quad \epsilon_{2}=\alpha E I-8 \sigma_{1} L_{0}^{3}>0, \\
\epsilon_{3}= & \beta c_{1}-\alpha \rho_{1}-\frac{\gamma c_{1} L}{2}-\frac{\gamma}{2} \rho_{1}>0, \\
\epsilon_{4}= & \beta c_{2}-\alpha \rho_{2}-\frac{\gamma c_{2} L}{2}-\frac{\gamma}{2} \rho_{2}>0, \\
\epsilon_{5}= & \alpha T_{1}-\frac{\gamma}{2} T_{1}-\frac{\gamma c_{1} L}{2}-2 \sigma_{2} L>0, \\
\epsilon_{6}= & \alpha T_{2}-\frac{\gamma}{2} T_{2}-\frac{\gamma c_{2} L}{2}-2 \sigma_{3} L>0, \\
\epsilon_{7}= & \beta k_{1}>0, \\
\epsilon_{9}= & \frac{\sigma_{1}}{2 L_{0}}>0, \quad \epsilon_{8}=\beta k_{2}>0, \\
\epsilon_{11}= & \frac{\sigma_{3}}{2 L}>0, \\
\lambda_{3}= & \min \left(\frac{2 \epsilon_{1}}{\beta \rho}, \frac{2 \epsilon_{2}}{\beta E I}, \frac{2 \epsilon_{3}}{\beta \rho_{1}}, \frac{2 \epsilon_{4}}{\beta \rho_{2}}, \frac{2 \epsilon_{5}}{\beta T_{1}}, \frac{2 \epsilon_{6}}{\beta T_{2}}, \frac{2 \epsilon_{7}}{\beta m_{1}}, \frac{2 \epsilon_{8}}{\beta m_{2}},\right. \\
& \left.\frac{2 \epsilon_{9}}{\alpha c}, \frac{2 \epsilon_{10}}{\alpha c_{1}}, \frac{2 \epsilon_{11}}{\alpha c_{2}}, \frac{2 \epsilon_{12}}{\beta k_{p}}\right)>0 .
\end{aligned}
$$

Combining Ineqs. (31) and (35), we have

$\dot{\Theta}(t) \leq-\lambda \Theta(t)+\varepsilon$,

where $\lambda=\lambda_{3} / \lambda_{2}$. Subsequently, we have the following main result of this paper as follows. Integrating of Ineq. (36), we obtain

$\Theta(t) \leq\left(\Theta(0)-\frac{\varepsilon}{\lambda}\right) e^{-\lambda t}+\frac{\varepsilon}{\lambda} \leq \Theta(0) e^{-\lambda t}+\frac{\varepsilon}{\lambda} \in \mathcal{L}_{\infty}$.

Utilizing inequality (15) and the Poincaré inequality (18), we have

$$
\begin{aligned}
\frac{\beta}{16 L_{0}^{3}} E I[w]^{2} \leq & \frac{\beta}{2} E I \int_{0}^{L_{0}}\left[w^{\prime \prime}\right]^{2} d x+\frac{\beta}{2} k_{p}[w(0, t)]^{2} \\
& -\left(\frac{\beta}{2} k_{p}-\frac{\beta}{8 L_{0}^{3}} E I\right)[w(0, t)]^{2}
\end{aligned}
$$

We design $k_{p}$ satisfying $k_{p}-\frac{E I}{4 L_{0}^{3}} \geq 0$, and we further have

$$
\begin{aligned}
\frac{\beta}{16 L_{0}^{3}} E I[w]^{2} & \leq \frac{\beta}{2} E I \int_{0}^{L_{0}}\left[w^{\prime \prime}\right]^{2} d x+\frac{\beta}{2} k_{p}[w(0, t)]^{2} \\
& \leq \Theta_{1}(t)+\Theta_{2}(t) \leq \frac{1}{\lambda_{1}} \Theta(t) \in \mathcal{L}_{\infty} .
\end{aligned}
$$

Suitably rewritten the above inequality, uniformly bounded of $w$ can be obtained as

$|w| \leq \sqrt{\frac{16 L_{0}^{3}}{\beta \lambda_{1} E I}\left(\Theta(0) e^{-\lambda t}+\frac{\varepsilon}{\lambda}\right)} \leq D_{0}$, 
where the constant $D_{0}=\sqrt{\frac{16 L_{0}^{3}}{\beta \lambda_{1} E I}\left(\Theta(0)+\frac{\varepsilon}{\lambda}\right)}, \forall x \in\left[0, L_{0}\right]$. In addition, as $t$ trends to infinity, we have

$\lim _{t \rightarrow \infty}|w| \leq \sqrt{\frac{16 L_{0}^{3} \varepsilon}{\beta \lambda_{1} \lambda E I}}, \quad \forall x \in\left[0, L_{0}\right]$.

In a similar manner, from Inequality (14), we have

$\frac{\beta}{2 L} T_{1}\left[w_{1}+D_{1}\right]^{2} \leq \frac{\beta}{2} T_{1} \int_{-L}^{0}\left[w_{1}^{\prime}\right]^{2} d x \leq \Theta_{1}(t) \leq \frac{1}{\lambda_{1}} \Theta(t) \in \mathcal{L}_{\infty}$,

$\frac{\beta}{2 L} T_{2}\left[w_{2}-D_{2}\right]^{2} \leq \frac{\beta}{2} T_{2} \int_{-L}^{0}\left[w_{2}^{\prime}\right]^{2} d x \leq \Theta_{1}(t) \leq \frac{1}{\lambda_{1}} \Theta(t) \in \mathcal{L}_{\infty}$.

Then, we obtain $w_{1}+D_{1}$ and $w_{2}-D_{2}$ are uniformly bounded as follows

$\left|w_{1}+D_{1}\right| \leq \sqrt{\frac{2 L}{\beta \lambda_{1} T_{1}}\left(\Theta(0) e^{-\lambda t}+\frac{\varepsilon}{\lambda}\right)} \leq D$,

$\left|w_{2}-D_{2}\right| \leq \sqrt{\frac{2 L}{\beta \lambda_{1} T_{2}}\left(\Theta(0) e^{-\lambda t}+\frac{\varepsilon}{\lambda}\right)} \leq D$

where the constant $D=\sqrt{\frac{2 L}{\beta \lambda_{1} \min \left(T_{1}, T_{2}\right)}\left(\Theta(0)+\frac{\varepsilon}{\lambda}\right)}, \forall x \in[-L, 0]$. Moreover, as $t$ trends to infinity, we have

$\lim _{t \rightarrow \infty}\left|w_{1}+D_{1}\right| \leq \sqrt{\frac{2 L \varepsilon}{\beta \lambda_{1} \lambda T_{1}}}$,
$\lim _{t \rightarrow \infty}\left|w_{2}-D_{2}\right| \leq \sqrt{\frac{2 L \varepsilon}{\beta \lambda_{1} \lambda T_{2}}}$,

$\forall x \in[-L, 0]$.

Remark 7. For this FWT system, the value of system parameters $E I$, $T_{1}, T_{2}, L$ and $L_{0}$ are relative larger than positive constants $\alpha, \beta, \gamma$, and $\sigma_{1}-\sigma_{3}$. According to inequalities $\epsilon_{1}-\epsilon_{12}$, when the system parameters $E I, T_{1}, T_{2}, L, L_{0}, \rho, \rho_{1}, \rho_{2}, c, c_{1}$ and $c_{2}$ are determined, $\alpha, \beta, \gamma$ and $\sigma_{1}-\sigma_{3}$ can be chosen to be small. Further, the value of control gains $k_{1}, k_{2}$ and $k_{p}$ can be determined to make $\epsilon_{7}, \epsilon_{8}$, and $\epsilon_{12}$ hold.

Remark 8. From Eqs. (41), (46) and (47), it is easy to know that the system's displacement $w, w_{1}$ and $w_{2}$ can converge to bounded range. Therefore, we can obtain that proposed control laws (19) and (20) are effective for suppressing vibrations of the FWT system.

Remark 9. All of the required information in the two controllers can be received by a backward difference method or obtained through a series of sensors. Using the laser displacement one can get $w_{L_{0}}$ and $w_{0}$, which are the tip boundary of the tower and the bottom base for the floating tower respectively (Queiroz, Dawson, Nagarkatti, \& Zhang, 2000). $\dot{w}_{L_{0}}$ and $\dot{w}_{0}$ with only one time derivative, which can be obtained by applying the backward difference algorithm.

\section{Numerical simulations}

In this part, we make numerical simulations to validate the effectiveness of proposed vibration control method for the FWT system. The dynamical behavior of the FWT system is analyzed in the set $\Omega=\left\{\left(x_{0}, x_{1}, t\right): 0 \leq x_{0} \leq L_{0}, 0 \leq x \leq L, 0 \leq t \leq T\right\}$. The temporal step size $k$ and spacial step sizes $h_{0}$ and $h$ as below

$$
\begin{aligned}
h_{0} & =\Delta x_{0}=\frac{L_{0}}{M_{0}}, \\
h & =\Delta x=\frac{L}{M} \\
k & =\Delta t=\frac{T}{N}
\end{aligned}
$$

where, $M_{0}, M$ and $N$ are the spacial subdivisions of the length of tower and mooring lines, and the time subdivisions, respectively. Referring for previous works (He \& Ge, 2015; Lyu, Zhang, \& Li, 2019; Musial,
Table 1

Parameters of the FWT system.

\begin{tabular}{llll}
\hline Parameter & Value & Parameter & Value \\
\hline$L_{0}$ & $20 \mathrm{~m}$ & $\rho$ & $10 \mathrm{~kg} / \mathrm{m}$ \\
$E I$ & $2000 \mathrm{Nm}^{2}$ & $c$ & $8 \mathrm{Ns} / \mathrm{m}^{2}$ \\
$L$ & $100 \mathrm{~m}$ & $\rho_{1}, \rho_{2}$ & $1.5 \mathrm{~kg} / \mathrm{m}$ \\
$T_{1}, T_{2}$ & $10^{4} \mathrm{~N}$ & $c_{1}, c_{2}$ & $0.8 \mathrm{Ns} / \mathrm{m}$ \\
$m_{1}$ & $150 \mathrm{~kg}$ & $m_{2}$ & $100 \mathrm{~kg}$ \\
$D_{1}$ & $5 \mathrm{~m}$ & $D_{2}$ & $5 \mathrm{~m}$ \\
\hline
\end{tabular}

Butterfield, \& Boone, 2004; Ruzzo, Saha, \& Arena, 2019; Wang, Hu, \& Meng, 2018), system parameters of the FWT system is given in Table 1 , and disturbances are given as $d_{1}(t)=[10+\sin (5 \pi t)] \times 10^{3} \mathrm{~N}$ and $d_{2}(t)=[2+3 \cos (10 \pi t)] \times 10^{4} \mathrm{~N}$.

Initial conditions of the FWT system are given as $w(x, 0)=\frac{x}{L_{0}} \mathrm{~m}$, $w_{1}(x, 0)=\sqrt{\frac{D_{1}^{2} x}{L}}-D_{1} \mathrm{~m}, w_{2}(x, 0)=-\sqrt{\frac{D_{2}^{2} x}{L}}+D_{2} \mathrm{~m}$, and $\dot{w}(x, 0)=$ $\dot{w}_{1}(x, 0)=\dot{w}_{2}(x, 0)=0 \mathrm{~m} / \mathrm{s}$, the time duration $t_{f}=25$ seconds.

In the numerical simulation, three control cases will be taken into consideration, namely, without control, with PD control, and with proposed vibration control.

Without control: In this case, there is no control input acting on the FWT system, namely, $u_{1}(t)=0, u_{2}(t)=0$. Fig. 2 illustrates the movements of the FWT system, where, the red thick line represents the motion of the tower, and the two blue lines describe the motion of two mooring lines. From Fig. 2, it is obvious that there exist vibrations in the flexible wind turbine tower and mooring lines under the effect of external disturbances, and the vibrations of FWT system are increasing with increasing time from 0 to $25 \mathrm{~s}$. Moreover, three-dimensional Figs. 5, 8 and 11 illustrate displacements of the turbine tower and mooring lines. It is also seen that increasing structural vibrations of FWT system have a great influence on system performance, even will damage the flexible structures. Therefore, it is necessary to propose vibration control to stabilize the FWT system.

PD control: In Fig. 3, the control performance for suppressing the vibration of FWT system of PD control is shown, where, PD controllers are given as $u_{1}(t)=5000 \dot{w}_{L_{0}}+1000 w_{L_{0}}, u_{2}(t)=5000 \dot{w}_{0}+1000 w_{0}$. From these figures, it can be seen that vibrations of the FWT system are reduced, and the vibration reduction for each mooring lines and tower are shown in Figs. 6, 9 and 12, respectively. According to these figures, we can see that there still exist small vibrations in turbine tower and mooring lines under PD control, which implies that PD control has a positive effect on the vibration suppression, while the control performance is not enough. Therefore, it is necessary to develop a better control scheme to reduce the vibration of FWT system. In Fig. 14, control inputs of the PD control are given. The input of controller $u_{1}(t)$ is in the range of $-1300 \mathrm{~N}$ to $1000 \mathrm{~N}$ from $0 s$ to $25 \mathrm{~s}$, and the input of controller $u_{2}(t)$ is in the range of $-2 \times 10^{4} \mathrm{~N}$ to $2 \times 10^{4} \mathrm{~N}$ from $0 s$ to $25 s$.

Active Boundary control: In this case, active boundary controllers $u_{1}(t)$ (19) and $u_{2}(t)$ (20) are implemented in the FWT system with a set of control parameters $k_{1}=5000, k_{2}=3000, k_{p}=800, \alpha=20, \beta=100$ and $\gamma=0.5$. Similar to the above cases, Fig. 4 illustrates the whole dynamics of FWT system, and from Fig. 4, we can see that vibrations of the FWT system are suppressed well. Moreover, the dynamics of each mooring lines and turbine tower are shown in Figs. 7, 10 and 13. Fig. 15 shows the input control signals of two proposed controllers: the input of controller $u_{1}(t)$ is in the range of $-1000 \mathrm{~N}$ to $1000 \mathrm{~N}$ from $0 \mathrm{~s}$ to $25 \mathrm{~s}$, and the input of controller $u_{2}(t)$ is in the range of $-3 \times 10^{4} \mathrm{~N}$ to $3 \times 10^{4} \mathrm{~N}$ from $0 s$ to $25 s$.

According to Figs. 3-13, it can be seen that the proposed active boundary controllers has a better control performance than PD control in suppressing the vibration of FWT system, where, vibrations of turbine tower and mooring lines have better convergence. Moreover, it is seen that the designed controllers are still able to reject the effect of the external disturbances and to ensure the FWT system stable. These simulations results are consensus with the theoretical results derived in (41), (46) and (47), and demonstrate that the proposed control laws are effective in vibration suppression for the FWT system. 

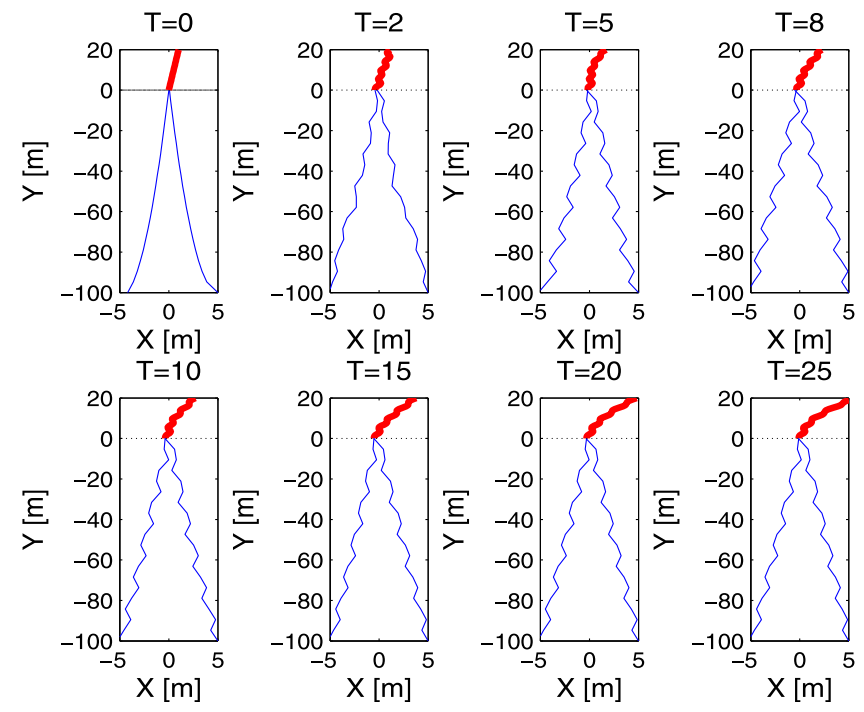

Fig. 2. Displacement of the FWT system: without control.
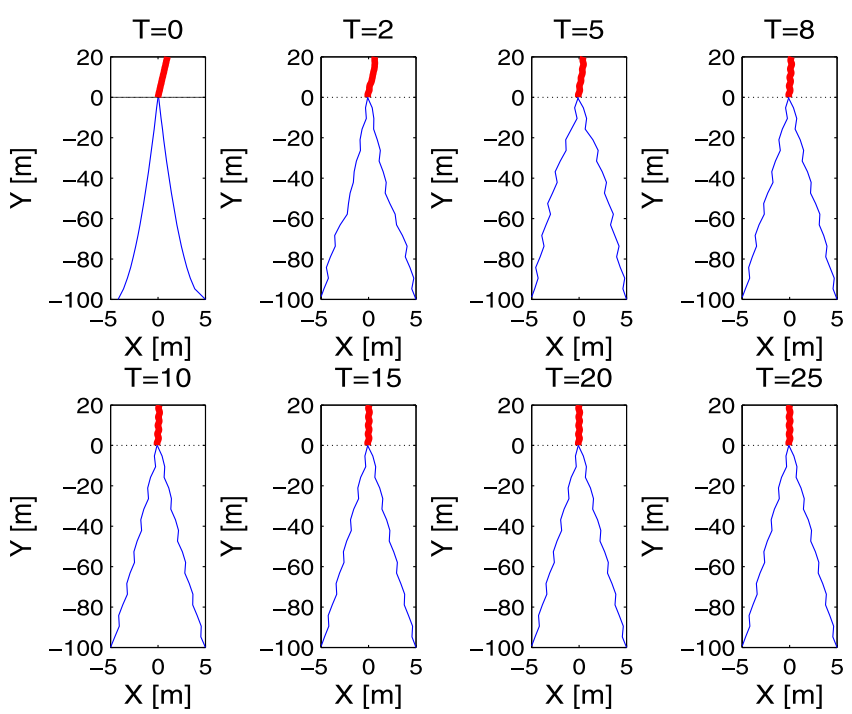

Fig. 3. Displacement of the FWT system movements: with the PD control.
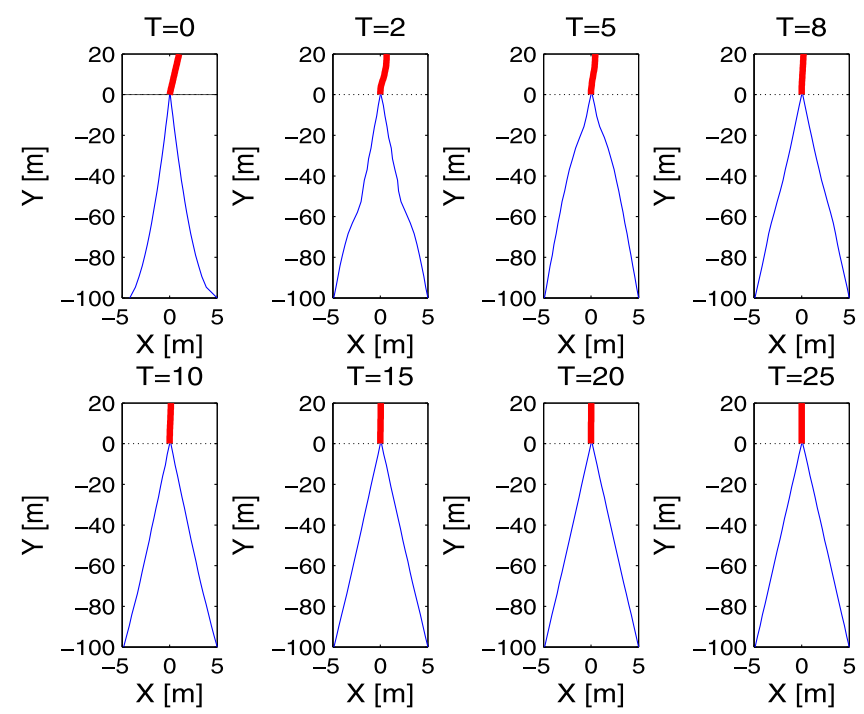

Fig. 4. Displacement of the FWT system movements: with the boundary control.

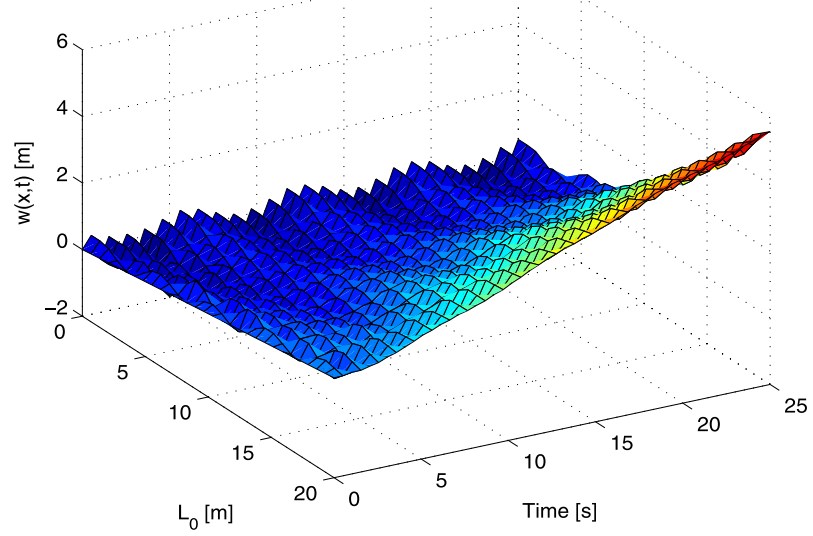

Fig. 5. $w(x, t)$, vibration of the tower without control.

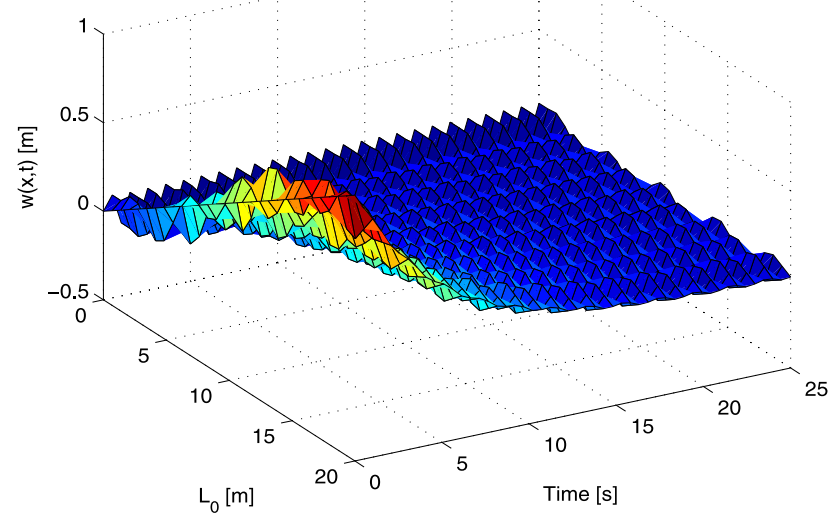

Fig. 6. $w(x, t)$, vibration of the tower with the PD control.

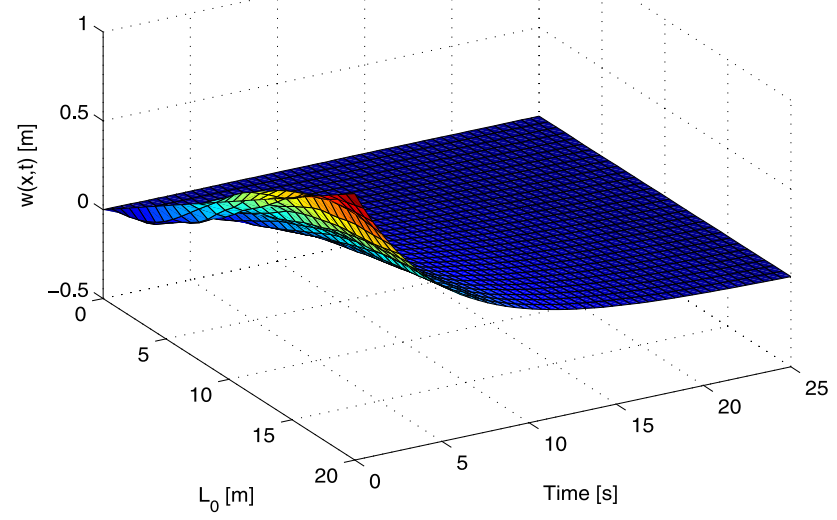

Fig. 7. $w(x, t)$, vibration of the tower with the proposed control.

\section{Conclusion}

In this paper, we address the stabilization problem of the FWT system, which is a beam-string coupled structure. Through proposing active vibration control method, vibrations of turbine tower and 


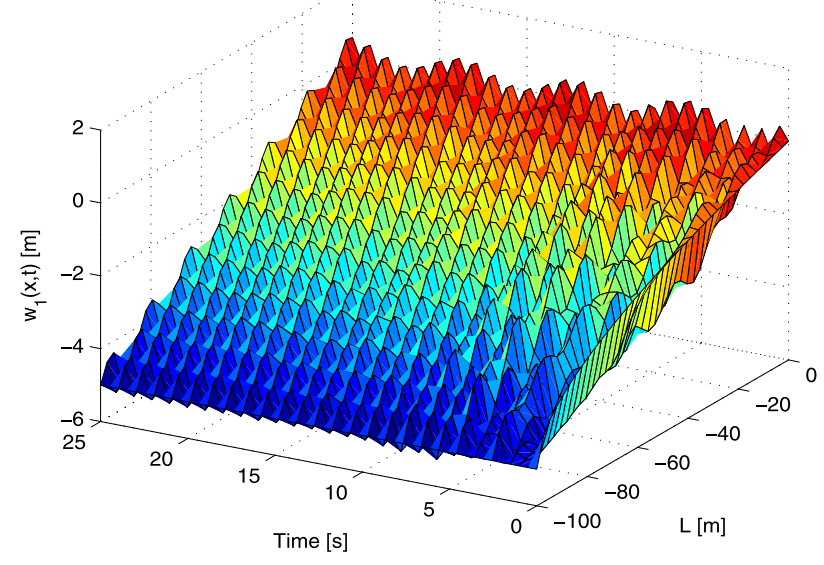

Fig. 8. $w_{1}(x, t)$, vibration of the left mooring line without control.

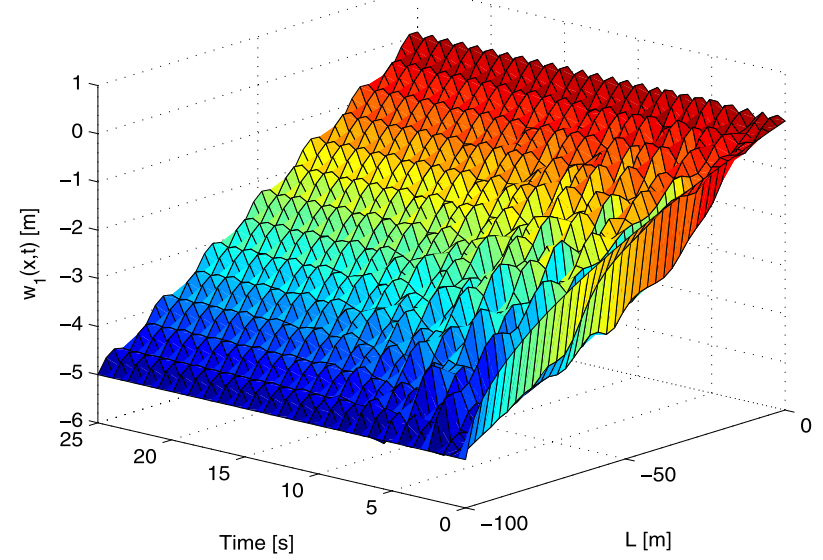

Fig. 9. $w_{1}(x, t)$, vibration of the left mooring line with PD control.

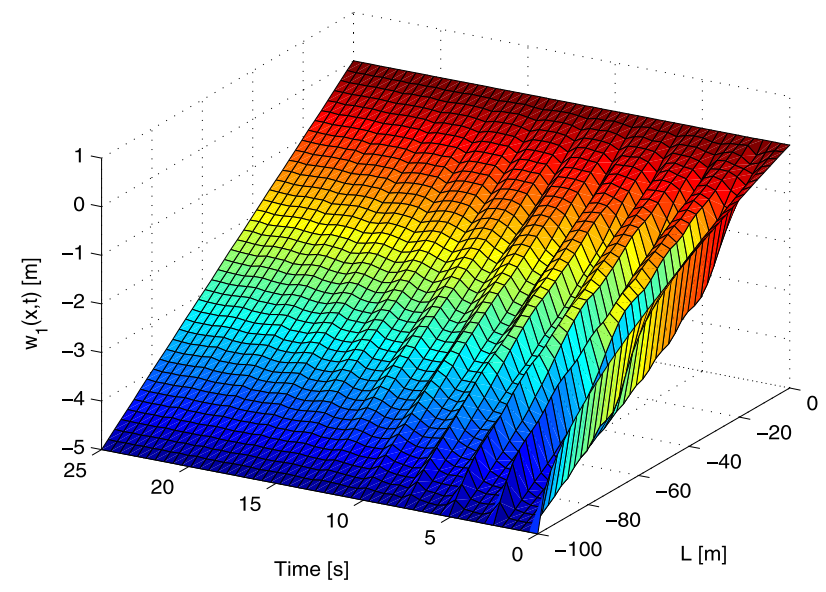

Fig. 10. $w_{1}(x, t)$, vibration of the left mooring line with the proposed control.

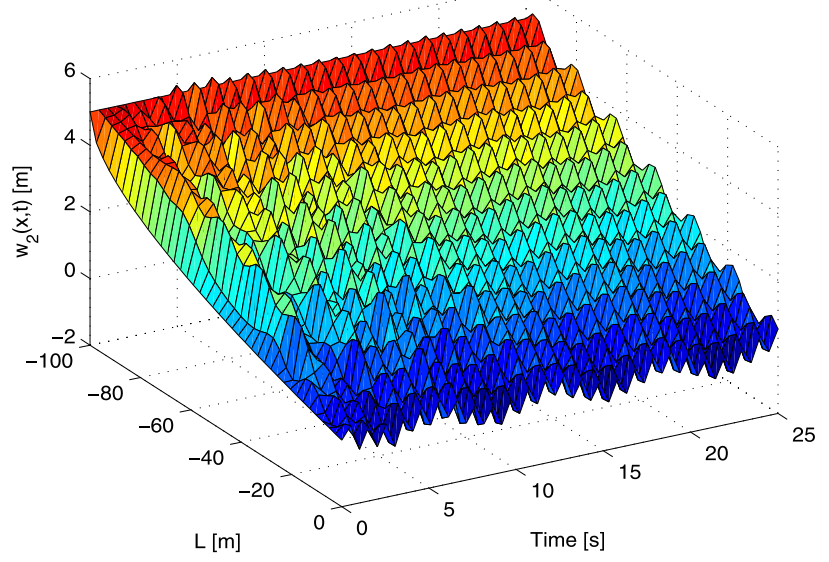

Fig. 11. $w_{2}(x, t)$, vibration of the right mooring line without control.

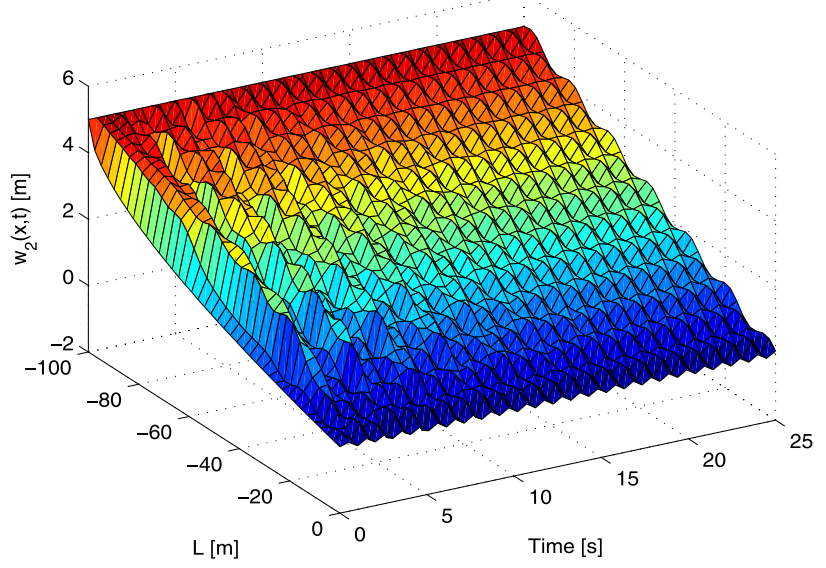

Fig. 12. $w_{2}(x, t)$, vibration of the right mooring line with PD control.

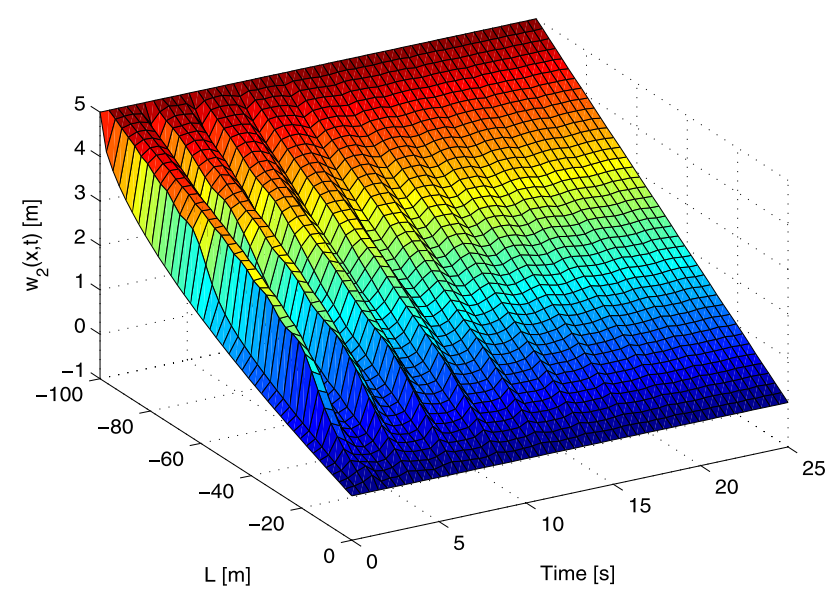

Fig. 13. $w_{2}(x, t)$, vibration of the right mooring line with the proposed control. 

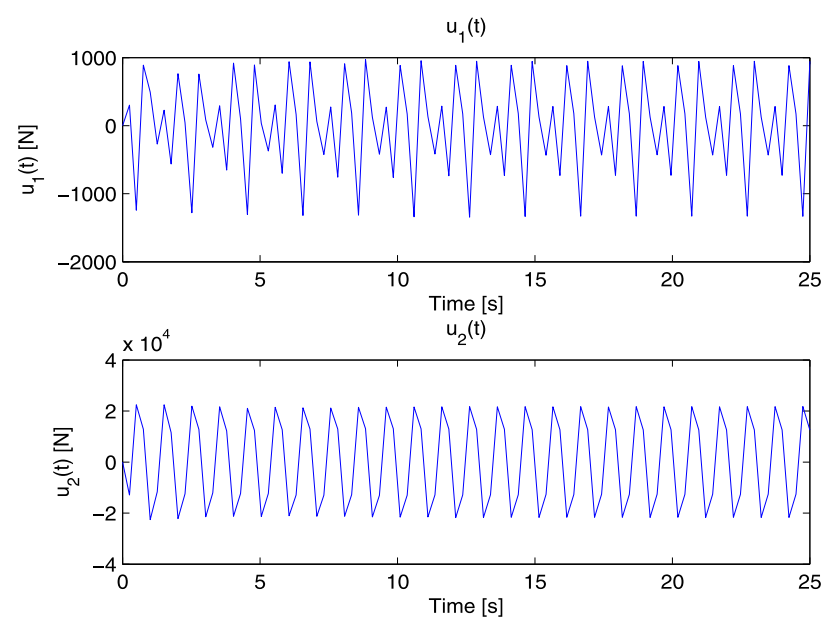

Fig. 14. PD control inputs..
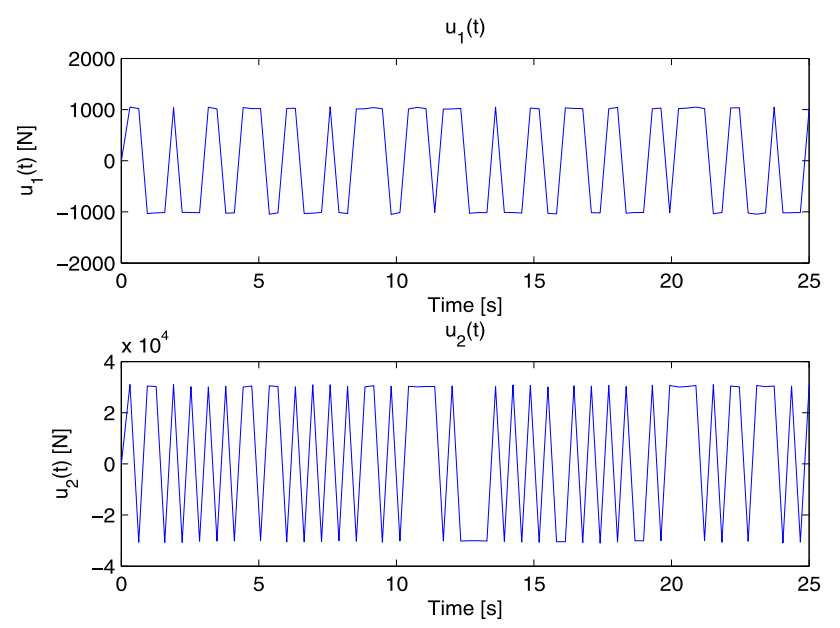

Fig. 15. Control inputs.

mooring lines can be suppressed obviously. Moreover, the stability of FWT system is also guaranteed by choosing a suitable Lyapunov function. Besides, the effectiveness of proposed controllers is validated by numerical simulation results. In future work, we will focus on the optimal control and finite-time vibration control to improve the control performances, e.g., rate of convergence, transient response, etc.

\section{Declaration of competing interest}

The authors declare that they have no known competing financial interests or personal relationships that could have appeared to influence the work reported in this paper.

\section{Acknowledgments}

The authors would like to thank the Editor-In-Chief, Associate Editor and anonymous reviewers for their constructive comments for improving the quality and presentation of this paper.

\section{References}

Ainsworth, T., \& Juneau, N. (2006). Significant wave height: A closer look at wave forecasts. NOAAs National Weather Service.

Bakka, T., \& Karimi, H. R. (2012). Robust dynamic output feedback control synthesis with pole placement constraints for offshore wind turbine systems. Mathematical Problems in Engineering, 2012(2).
Bakka, T., \& Karimi, H. R. (2013). $\mathrm{H}_{\infty}$ static output-feedback control design with constrained information for offshore wind turbine system. Journal of the Franklin Institute, 350(8), 2244-2260.

Bakka, T., Karimi, H.-R., \& Christiansen, S. (2014). Linear parameter-varying modelling and control of an offshore wind turbine with constrained information. IET Control Theory \& Applications, 8(1), 22-29.

Bhikkaji, B., Moheimani, S., \& Petersen, I. (2012). A negative imaginary approach to modeling and control of a collocated structure. IEEE/ASME Transactions on Mechatronics, 17(4), 717-727.

Chao, Z., Chenguang, Y., \& Zhaopeng, C. (2020). Bio-inspired robotic impedance adaptation for human-robot collaborative tasks. Science China. Information Sciences, http://dx.doi.org/10.1007/s11432-019-2748-x.

Chen, C.-C., \& Chang, J.-Y. (2013). Vision-assisted vibration analysis of inhomogeneous flexible cables in hard disk drives. IEEE Transactions on Magnetics, 49(6), 2628-2633.

Chen, M., Ge, S. S., How, B. V. E., \& Choo, Y. S. (2013). Robust adaptive position mooring control for marine vessels. IEEE Transactions on Control Systems Technology, 21(2), 395-409.

Chen, M., Shao, S.-Y., \& Jiang, B. (2017). Adaptive neural control of uncertain nonlinear systems using disturbance observer. IEEE Transactions on Cybernetics, 47(10), 3110-3123.

Dai, S.-L., He, S., Wang, M., \& Yuan, C. (2018). Adaptive neural control of underactuated surface vessels with prescribed performance guarantees. IEEE Transactions on Neural Networks and Learning Systems, 30(12), 3686-3698.

De Domenico, D., \& Ricciardi, G. (2018). Optimal design and seismic performance of tuned mass damper inerter (TMDI) for structures with nonlinear base isolation systems. Earthquake Engineering \& Structural Dynamics, 47(12), 2539-2560.

Do, K. D. (2017). Stochastic boundary control design for extensible marine risers in three dimensional space. Automatica, 77(3), 184-197.

Endo, T., Sasaki, M., \& Matsuno, F. (2017). Contact-force control of a flexible timoshenko arm. IEEE Transactions on Automatic Control, 62(2), 1004-1009.

Endo, T., Sasaki, M., Matsuno, F., \& Jia, Y. (2016). Contact-force control of a flexible timoshenko arm in rigid/soft environment. IEEE Transactions on Automatic Control, 62(5), 2546-2553.

Fu, B., Jiang, H., \& Wu, T. (2019). Comparative studies of vibration control effects between structures with particle dampers and tuned liquid dampers using substructure shake table testing methods. Soil Dynamics and Earthquake Engineering, 121, 421-435.

Goldstein, H. (1951). Classical mechanics. Massachusetts, USA: Addison-Wesley.

Guo, B.-Z., \& Jin, F.-F. (2015). Output feedback stabilization for one-dimensional wave equation subject to boundary disturbance. IEEE Transactions on Automatic Control, 60(3), 824-830.

He, W., \& Ge, S. S. (2015). Vibration control of a nonuniform wind turbine tower via disturbance observer. IEEE/ASME Transactions on Mechatronics, 20(1), 237-244.

He, W., Ge, S. S., \& Huang, D. (2015). Modeling and vibration control for a nonlinear moving string with output constraint. IEEE/ASME Transactions on Mechatronics, 20(4), 1886-1897.

He, X., He, W., Liu, Y., Wang, Y., Li, G., \& Wang, Y. (2018). Robust adaptive control of an offshore ocean thermal energy conversion system. IEEE Transactions on Systems, Man, and Cybernetics: Systems, http://dx.doi.org/10.1109/TSMC.2018.2870999.

He, X., He, W., Shi, J., \& Sun, C. (2017). Boundary vibration control of variable length crane systems in two-dimensional space with output constraints. IEEE/ASME Transactions on Mechatronics, 22(5), 1952-1962.

He, W., He, X., \& Sun, C. (2017). Vibration control of an industrial moving strip in the presence of input deadzone. IEEE Transactions on Industrial Electronics, 64(6), 4680-4689.

He, W., Zhang, S., \& Ge, S. S. (2014). Robust adaptive control of a thruster assisted position mooring system. Automatica, 50(7), 1843-1851.

Holthuijsen, L. H. (2007). Waves in oceanic and coastal waters. Cambridge University Press.

Huang, D., Xu, J.-X., Li, X., Xu, C., \& Yu, M. (2013). D-type anticipatory iterative learning control for a class of inhomogeneous heat equations. Automatica, 49(8), 2397-2408.

Jin, F.-F., \& Guo, B.-Z. (2015). Lyapunov approach to output feedback stabilization for the Euler-Bernoulli beam equation with boundary input disturbance. Automatica, 52(1), 95-102.

Lamas, P. M., \& Fernandez, R. P. (2011). Floating offshore wind farms: The seed of artificial. Journal of Marine Technology and Environment, 2, 95-106.

Li, Z., Chen, Z., Fu, J., \& Sun, C. (2016). Direct adaptive controller for uncertain MIMO dynamic systems with time-varying delay and dead-zone inputs. Automatica, 63, 287-291.

Li, L., Chen, Z., Wang, Y., Zhang, X., \& Wang, N. (2019). Robust task-space tracking for free-floating space manipulators by cerebellar model articulation controller. Assembly Automation, 39(1), 26-33.

Li, X., \& Gao, H. (2016). Load mitigation for a floating wind turbine via generalized $H_{\infty}$ structural control. IEEE Transactions on Industrial Electronics, 63(1), 332-342.

Li, Z., Huang, B., Ajoudani, A., Yang, C., Su, C.-Y., \& Bicchi, A. (2017). Asymmetric bimanual control of dual-arm exoskeletons for human-cooperative manipulations. IEEE Transactions on Robotics, 34(1), 264-271. 
Li, H., Wang, L., Du, H., \& Boulkroune, A. (2016). Adaptive fuzzy backstepping tracking control for strict-feedback systems with input delay. IEEE Transactions on Fuzzy Systems, 25(3), 642-652.

Liu, Z., He, X., Zhao, Z., Ahn, C. K., \& Li, H.-X. (2020). Vibration control for spatial aerial refueling hoses with bounded actuators. IEEE Transactions on Industrial Electronics, http://dx.doi.org/10.1109/TIE.2020.2984442.

Lyu, G., Zhang, H., \& Li, J. (2019). Effects of incident wind/wave directions on dynamic response of a SPAR-type floating offshore wind turbine system. Acta Mechanica Sinica, 35(5), 954-963.

Ming, W., Yanlu, Z., Huifang, D., \& Junzhi, Y. (2020). Trajectory tracking control of a bionic robotic fish based on iterative learning. Science China. Information Sciences, http://dx.doi.org/10.1007/s11432-019-2760-5.

Moheimani, S., Pota, H., \& Petersen, I. (1999). Spatial balanced model reduction for flexible structures. Automatica, 35(2), 269-277.

Musial, W., Butterfield, S., \& Boone, A. (2004). Feasibility of floating platform systems for wind turbines. In 42nd AIAA aerospace sciences meeting and exhibit (p. 1007).

Nguyen, Q. C., \& Hong, K.-S. (2010). Asymptotic stabilization of a nonlinear axially moving string by adaptive boundary control. Journal of Sound and Vibration, 329(22), 4588-4603.

Nguyen, Q. C., \& Hong, K.-S. (2012). Simultaneous control of longitudinal and transverse vibrations of an axially moving string with velocity tracking. Journal of Sound and Vibration, 331(13), 3006-3019.

Pota, H. R., \& Alberts, T. E. (1995). Multivariable transfer functions for a slewing piezoelectric laminate beam. Journal of Dynamic Systems, Measurement, and Control, 117(3), 352-359.

Queiroz, M. S., Dawson, D. M., Nagarkatti, S. P., \& Zhang, F. (2000). Lyapunov based control of mechanical systems. Boston, USA: Birkhauser.

Ren, Y., Chen, M., \& Liu, J. (2020). Bilateral coordinate boundary adaptive control for a helicopter lifting system with backlash-like hysteresis. Science China. Information Sciences, http://dx.doi.org/10.1007/s11432-018-9636-3.

Ren, B., Wang, J.-M., \& Krstic, M. (2013). Stabilization of an ODE-Schrödinger cascade. Systems \& Control Letters, 62(6), 503-510.

Ruzzo, C., Saha, N., \& Arena, F. (2019). Wave spectral analysis for design of a spar floating wind turbine in mediterranean sea. Ocean Engineering, 184, 255-272.

Si, Y., Karimi, H. R., \& Gao, H. (2013). Modeling and parameter analysis of the OC3hywind floating wind turbine with a tuned mass damper in nacelle. Journal of Applied Mathematics, 2013(4), 1-10.

Smyshlyaev, A., \& Krstic, M. (2010). Adaptive control of parabolic PDEs. New Jersey, USA: Princeton University Press.
Stewart, G., \& Lackner, M. (2013). Offshore wind turbine load reduction employing optimal passive tuned mass damping systems. IEEE Transactions on Control Systems Technology, 21(4), 1090-1104.

Sun, C., \& Xia, Y. (2009). An analysis of a neural dynamical approach to solving optimization problems. IEEE Transactions on Automatic Control, 54(8), 1972-1977.

Tee, K. P., \& Ge, S. S. (2006). Control of fully actuated ocean surface vessels using a class of feedforward approximators. IEEE Transactions on Control Systems Technology, 14(4), 750-756.

Tong, X., Zhao, X., \& Zhao, S. (2017). Load reduction of a monopile wind turbine tower using optimal tuned mass dampers. International Journal of Control, 90(7), 1283-1298.

Wang, H., Hu, Z.-Q., \& Meng, X.-Y. (2018). Dynamic performance investigation of a spar-type floating wind turbine under different sea conditions. China Ocean Engineering, 32(3), 256-265.

Wang, J.-M., Liu, J.-J., Ren, B., \& Chen, J. (2015). Sliding mode control to stabilization of cascaded heat PDE-ODE systems subject to boundary control matched disturbance. Automatica, 52, 23-34.

Wang, J.-M., Ren, B., \& Krstic, M. (2012). Stabilization and Gevrey regularity of a Schrödinger equation in boundary feedback with a heat equation. IEEE Transactions on Automatic Control, 57(1), 179-185.

Van der Woude, C. (2011). A study on vibration isolation in a wind turbine subjected to wind and seismic loading (Master thesis), University of Waterloo.

Wu, G., Sun, J., \& Chen, J. (2018). Optimal linear quadratic regulator of switched systems. IEEE Transactions on Automatic Control, 64(7), 2898-2904.

Wu, H.-N., \& Wang, J.-W. (2014). Static output feedback control via PDE boundary and ODE measurements in linear cascaded ODE-beam systems. Automatica, 50(11), 2787-2798.

Wu, H.-N., Wang, J.-W., \& Li, H.-X. (2012). Design of distributed $H_{\infty}$ fuzzy controllers with constraint for nonlinear hyperbolic PDE systems. Automatica, 48(10), 2535-2543.

Xie, G., Sun, L., Wen, T., Hei, X., \& Qian, F. (2019). Adaptive transition probability matrix-based parallel IMM algorithm. IEEE Transactions on Systems, Man, and Cybernetics: Systems, http://dx.doi.org/10.1109/TSMC.2019.2922305.

Zhang, Z., Basu, B., \& Nielsen, S. R. (2019). Real-time hybrid aeroelastic simulation of wind turbines with various types of full-scale tuned liquid dampers. Wind Energy, 22(2), 239-256.

Zhang, S., Yang, C., Nie, S., \& He, W. (2015). Modeling and vibration control of a coupled floating wind turbine system. In Proceedings of the 2015 15th international conference on control, automation and systems, Busan, Korea (pp. 1989-1994). 